\title{
Transition to Adult Health Care for Adolescents and Young Adults with Cancer
}

\author{
David S. Rosen, M.D., M.P.H.
}

\begin{abstract}
As the prognosis for children and adolescents with cancer improves, we must reconsider how we will provide care to the adolescents and young adults who have survived these conditions. On the premise that the global needs of adults are best served by health care obtained in the adult health-care system, the case is made for transition from pediatric to adult-oriented health care for adolescents and young adults with cancer. The benefits of transition, barriers to implementation, and models of care are discussed. Cancer 1993; 71:3411-4.
\end{abstract}

Key words: adolescence, transition, chronic illness, health-care services.

The prognosis for children and adolescents with cancer, like that for children and adolescents with other chronic illnesses and disabilities, has improved considerably over the past 2 decades. ${ }^{1}$ For some malignancies, we now anticipate complete cure; for others, the probability of long-term survival has significantly increased. Dramatic improvements in outcome, however, have not been accompanied by equal progress in the manner in which we provide care to adolescents and young adults with these conditions. Recently, health-care providers have begun to fully appreciate the importance of addressing the unique needs of adolescents with chronic conditions. Problems of adjustment, limited opportunities for socialization, perturbations of growth and sexual maturation, and other medical and psychosocial issues are being explored in the medical literature. Even financial, educational, and vocational issues are beginning to receive much needed attention from

Presented at the American Cancer Society Workshop on Children with Cancer, Naples, Florida, September 5-6, 1991.

From the University of Michigan Medical School, Ann Arbor, Michigan.

The author thanks Nancy Okinow and Gayle Geber of the National Center for Youth with Disabilities for their invaluable collaboration.

Address for reprints: David S. Rosen, M.D., M.P.H., Department of Pediatrics, University of Michigan, Room 1924 Taubman Center, Box 0318, Ann Arbor, MI 48109-0318

Accepted for publication May 14, 1992. health-care providers. ${ }^{2}$ Missing from the discussion, however, has been the acknowledgement that at some point in time, adolescence ends and adulthood begins for all young people, even those with special healthcare needs. Missing too has been the recognition that for these young people, transition from child-centered to adult-oriented health care must occur and that this transfer will require special attention.

To many, the transition from pediatric to adult-oriented health care for those with chronic conditions, with the implied lapse in continuity of care, seems counterproductive. Others have been more assertive in their contention that care for adolescents and young adults with special needs be consolidated within pediatrics. ${ }^{3}$ Nevertheless, the benefits of this transition are substantial. They are as follows:

1. A Sense of Future. Ongoing child-centered care effects the subtle message that adulthood itself may be an unrealistic expectation. In contrast, transition to adulthood and adult health care suggests that the young person with cancer in fact has a future. From the practitioner, it is a statement of confidence and optimism; for the young person and their family, it is a positive statement about commitment to living and striving to reach one's full potential. A positive sense of the future has been associated with a greater sense of control in adolescent patients with cancer, which in turn helps to support emotional well-being. ${ }^{4}$

2. A Valued Member of Society. A move to adult health care is a marker for overall trarsition to adulthood. Moving into adult roles signals to young people that they are valued members of society who are expected to participate and contribute as adults. Loss of independence and dependence on adults are among the major fears of adolescents with cancer. ${ }^{5}$ Obtaining health care as an adult encourages young people to emancipate themselves from dependent family relationships, to be self-reliant in their care, and to advocate successfully for themselves. Success at self-care enhances self-es- 
teem, improves compliance, and may limit emotional regression, risk-taking behaviors, or other self-destructive responses to illness. ${ }^{6}$

3. Age-Appropriate Health Care. An appropriate transition to adult-centered health care for adolescents and young adults with cancer assures that their overall health-care needs will still be met as they become adults. Young adults need health care that is age-appropriate; adult-oriented practitioners, not pediatricians, are best trained to work with adult patients. Careful planning for transitional health care will also help to ensure that the unique health needs of adolescents are addressed.

4. Ongoing Medical Surveillance. When an appropriate transition to the adult health care sector is not provided, there is the real risk that patients will be lost to follow-up should they unilaterally decide that they have outgrown pediatric care. Also, compliance with appointment-keeping may decline when patients begin to feel uncomfortable in the pediatric setting. A formal transition plan anticipates and obviates these concerns by proactively introducing patients to the adult health-care system. Continuity of care is maintained through formal or informal linkages between the pediatric providers and the new adult-oriented providers.

A standard prescription for transition to adult health care is a difficult one to design. Both the timing and structure of transition must be determined on an individual basis. Some patients may be prepared for transition in their teenage years, and others may be best served by continuing in the pediatric setting well into the third decade of life. For some, transition will proceed quickly and smoothly; for others, transition will be a protracted or problematic experience.

Transition to adult-oriented health care for young people with cancer will be most successful if certain principles and features are incorporated into its planning. Foremost, all care should be based on the premise that transition to adult care will eventually occur. It is unrealistic to expect adolescents, their families, or health-care providers to accept transition if it is introduced with little warning or in times of crisis. The concept of transition should therefore be introduced to patients and their families early. This allows all participants to become accustomed to the idea that care will at some time be provided in a different setting.

Preparation for transition should specifically include health education and training in self-care. Although adequate knowledge does not ensure good health habits, lack of information or misinformation will almost certainly interfere with treatment plans. Specific information about a young person's disease pro- cess should be provided in a form that is appropriate to the expanding cognitive and developmental abilities of the individual. Moreover, such information should be directed to the adolescent (rather than to parents), be youth-oriented, and reflect the interests and concerns of adolescents and young adults. Pfefferbaum and Levenson have shown that adolescents with cancer differ significantly from their caregivers on the issues most important to them. ${ }^{7}$ Health information should be presented as the cornerstone of more independent care. To prepare them for the role of adult health-care consumer, adolescents with cancer should be encouraged to participate as soon as possible and as fully as possible in their own care. They should receive understandable explanations of diagnostic and therapeutic procedures and protocols, should participate in the decisions regarding diagnostic investigations, treatment regimens, and other care, and should be asked, along with their parents, to consent to medical and surgical procedures. Adolescents should be helped to gradually take on the health-care responsibilities previously assumed by their families and to become increasingly independent in meeting their daily needs. Health-care providers should be willing to negotiate care with their adolescent patients and to place greater emphasis on their preferences and lifestyles. In some cases, the physician will need to accept a "suboptimal" plan for care when the alternative is an "optimal" treatment plan that the adolescent finds unrealistic, excessive, or aversive and therefore ignores. Health-care providers must accept that multiple approaches exist for any given problem and be willing to trade their concept of ideal care for an acceptable alternative that acknowledges the priorities and desires of the adolescent. ${ }^{8}$

When transition does occur, care in the adult setting must be equivalent in both the quality and intensity of services. The new setting will be immediately unacceptable to both young people and their families if it represents a decline in the level of care to which they have become accustomed. It is equally important to recognize that transition is a process, not an event. During the period of transition, young people and their families should not be expected to completely abandon the security of their familiar pediatric health care. New adultoriented providers should be introduced before they assume the responsibility of care coordination. Although it may not be possible in all settings, a period of concurrent care, where the pediatric and adult-oriented teams work together, is ideal. Both the pediatric and adult-oriented provider should be involved in planning for transition and monitoring its progress. Ongoing assessment and midcourse adjustments allow the health-care providers to respond to changes in health status, care needs, or functional ability. 
Because adolescence is variable in its progression, transition to adult health-care services must be individually timed. Adolescents experience physical maturation, emerging sexuality, and must establish a satisfactory body image. They are expected to achieve greater psychologic and cognitive sophistication and to become part of a peer group. Eventually, adolescents should become less dependent on familial support, conform less to peer norms, and develop the capacity for intimate relationships. Adolescence concludes once these developmental tasks are complete and a stable identity is formed. For all adolescents, the progress and tempo of this process varies; it may be especially protracted or difficult when the adolescent is further stressed by a serious illness or chronic condition. Thus, there is no clear endpoint to adolescence and no simple formula for determining transition readiness. It falls to the health-care providers to determine an appropriate time for transition based on criterion such as medical status, developmental milestones, knowledge of the disease process, and success at self-care.

Finally, movement to the adult health-care sector should explicitly include plans for primary care and overall care coordination. Too often, those with chronic conditions get exemplary care for their specific illness but little or no preventative health care or health supervision. $^{9,10}$ Indicated health screening may not be performed, immunizations may be forgotten, and psychosocial health may be neglected. For example, although we do not expect to find excess psychopathology in the adolescent and young adult survivors of childhood cancer, ${ }^{11-13}$ adjustment issues related to sexuality and relationships have been found. The majority of these had not been identified or addressed. ${ }^{12}$ As transition proceeds and care is extended throughout the pediatric and adult-oriented health-care systems, a single identifiable care coordinator becomes even more crucial to ensure that global health needs are met.

Inertia has perhaps been the greatest barrier to creating opportunities for transition for young people with chronic conditions. Few are anxious to tamper with a successful program of care. There is little enthusiasm among young people or their families to abandon providers who have served them well. This might be especially true for persons with cancer who have received life-saving therapy yet remain at risk for recurrence. Pediatric health-care providers are no more ready to sever their relationship with patients. They are interested in identifying recurrences or second malignancies, in following the late physiologic effects of previous treatment, and in the psychosocial status of their patients later in life. Often, they will have established close ties to their patients and be unwilling to see these threatened. For their part, adult-oriented oncologists may be less familiar with childhood malignancies and feel less competent to follow their survivors. Financial issues may intrude into the transition process; inability to obtain health insurance may make any changes in care problematic. Finally, when care needs are great, simply negotiating the activities of daily living may compound what Hull called the "difficulty in recognizing the need for change in a context of chronicity."14

\section{Models of Transition}

The issue of transition from child-centered to adult-oriented health care is a pressing one but one that has been poorly addressed in the medical literature. Schidlow and Fiel have described a transition program for adolescents with cystic fibrosis. ${ }^{15}$ In their model, "transition clinics" allow the graded transfer of care from pediatric to adult-oriented specialists. For a time, care is provided concurrently; eventually, complete transition to the adult setting occurs. This is simply one among many possible approaches. Hospital-based inpatient programs, informal linkages between pediatric and adult-oriented providers, or interventions directed by specialists in adolescent medicine may all be appropriate in different settings. In general, however, models of transitional care have been neither well described nor adequately evaluated, and there is little guidance available to aid in the design of appropriate interventions. The interdisciplinary approach to care, common in the pediatric environment, may prove to be equally productive in adult health-care settings.

Many issues remain to be resolved before healthcare transition can become commonplace for adolescents and young adults with cancer and other special health-care needs. Health-care providers in the adult sector will need to prepare themselves to care for the what were once thought of as diseases of childhood. This will require educational effort from pediatricians and from adult physicians, a willingness to learn. Adult-based health-care services for those with special needs must expand and reach out to young adults. Financial, administrative, and institutional impediments must be addressed, and a mechanism must be devised to ensure that medical information and records can be relocated efficiently. Most importantly, a fundamental change in attitude is required, one that acknowledges that adults are best cared for in an environment designed for adults.

The status quo is slowly changing as both professional and consumer groups recognize the need and advocate for appropriate transition to adult-oriented care. ${ }^{16-21}$ Health-care transition challenges young people, their families, and their health-care providers. Meeting that challenge rewards us all with empowered 
young people who participate fully in their own health care and in their lives.

\section{References}

1. Gortmaker SL, Sapprfield W. Chronic childhood disorders: prevalence and impact. Pediatr Clin North Am 1984; 31:3-18.

2. Mellette SJ, Franco PC. Psychosocial barriers to employment of the cancer survivor. I Psychosoc Oncol 1987; 5:97-115.

3. Garson $A$. The science and practice of pediatric cardiology in the next decade. Am Heart J 1987; 114:462-8.

4. Nannis ED, Susman EJ, Strope BE, Woodruff PJ, Hersh SP, Levine AS, et al. Correlates of control in pediatric cancer patients and their families. J Pediatr Psychol 1982; 7:75-84.

5. Delengowski A, Dugan-Jordan M. Care of the adolescent cancer patient on an adult medical oncology unit. Semin Oncol Nurs $1986 ; 2: 95-103$.

6. Zeltzer LK. The adolescent with cancer. In: Kellerman J, editor. Psychological aspects of childhood cancer. Springfield, IL: Charles C. Thomas, 1980:70-99.

7. Pfefferbaum B, Levenson PM. Adolescent cancer patient and physician responses to a questionnaire on patient concerns. $A m$ I Psychiat 1982; 139:348-51.

8. Coupey SM, Cohen MI. Special considerations for the health care of adolescents with chronic illness. Pediatr Clin North Am 1984; 31:211-9.

9. Palfrey JS, Levy JC, Gilbert KL. Use of primary care facilities by patients attending specialty clinics. Pediatrics $1980 ; 65: 567-72$.

10. Carrol G, Massarelli E, Opzoomer A, Pekeles G, Pedneault M, Frappier J, et al. Adolescents with chronic disease: are they receiving comprehensive care? / Adolescent Health Care 1983; $4: 261-5$.
11. Fritz GK, Williams JR, Amylon M. After treatment ends: psychosocial sequelae in pediatric cancer survivors. Am J Orthopsychiatr $1988 ; 58: 552-61$.

12. Fritz GK, Williams JR. Issues of adolescent development for survivors of childhood cancer. I Am Acad Child Adolesc Psychiatry 1988; 27:712-5.

13. Tebbi CK, Mallon JC. Long-term psychosocial outcome among cancer amputees in adolescence and early adulthood. J Psychosucial Oncol 1987; 5:69-82.

14. Hull R. Care of physically disabled young adults. Br Med J 1988; 296(6638):1739.

15. Schidlow DV, Fiel SB. Life beyond pediatrics: transition of chronically ill adolescents from pediatric to adult health care systems. Med Clin North Am 1990; 74:1113-20.

16. Moore IM, Klopovich P. Future perspectives. Semin Oncol Nurs 1989; 5:70.

17. Barbero GJ. Leaving the pediatrician for the internist [editorial]. Ann Intern Med 1982; 96:673-4.

18. Cystic Fibrosis Center Committee and Guidelines Subcommittee. Cystic Fibrosis Foundation Guidelines for patient services, evaluation, and monitoring in cystic fibrosis centers. IAMA $1990 ; 144: 1311-2$.

19. Spina Bifida Association of America. 17th Annual Conference Proceedings, 1990.

20. Berg P. Youth in transition: developing appropriate services for adult survivors of pediatric disorders. Minneapolis: National Center for Youth with Disabilities, 1988.

21. Magrab PR, Millar HEC. Surgeon General's Conference: growing up and getting medical care: youth with special health care needs. Washington, DC: National Center for Networking Community Based Services, 1989. 